\title{
MRI Evaluation of Acute Internal Derangement of Knee
}

\author{
Venkateshwaran Arumugam1, Ganesan Ram Ganesan'2, Paarthipan Natarajan ${ }^{3}$ \\ ${ }^{1}$ Meenakshi Medical College and Research Institute, Kancheepuram, India \\ ${ }^{2}$ Sriramachandra Medical College and Research Institute, Chennai, India \\ ${ }^{3}$ Meenakshi Medical College and Research Institute, Kancheepuram, India \\ Email: venkateshwaran81@gmail.com
}

Received 19 April 2015; accepted 30 May 2015; published 2 June 2015

Copyright (C) 2015 by authors and Scientific Research Publishing Inc.

This work is licensed under the Creative Commons Attribution International License (CC BY).

http://creativecommons.org/licenses/by/4.0/

(c) (i) Open Access

\begin{abstract}
Introduction: Internal derangement of the knee is a blanket term used to cover a group of disorders involving destruction of the normal functioning of the ligaments or cartilages. MRI provides excellent soft tissue contrast and is capable of evaluating the soft tissue and bony structures in multiple imaging planes which provide significant advantage in IDK. Methods and Materials: A prospective study of hundred twenty patients who underwent MRI for the diagnosis of internal derangement of knee between the period January 2013 and January 2015 was conducted. All the patients gave a history of knee joint pain following trauma and clinically suspected to have meniscal and ligament tears. Patients were evaluated using Philips intera R 111.5 T with pulsar gradient system using a sensor extremity coil. Results: Commonest lesion detected in our study was ACL tear followed by meniscal tear and medial collateral ligament injury. The most common sign of cruciate ligament injury was hyper intensity in the ligament. Grade 3 was the most common grade of meniscal tear. Conclusion: MRI is unique in its ability to evaluate the internal structure as well as the surface of the meniscus. Many anatomical variants can mimic a tear on MRI. MRI is an excellent non invasive modality for imaging the knee.
\end{abstract}

\section{Keywords}

Anterior Cruciate, Posterior Cruciate, Meniscus, Internal Derangement Knee

\section{Introduction}

Internal derangement of the knee is a blanket term used to cover a group of disorders involving destruction of the normal functioning of the ligaments or cartilages. IDK is evaluated with radiography, MRI, arthrography and 
arthroscopy. Arthroscopy is usually considered as a gold standard for the evaluation of knee injury. However, the accuracy of arthroscopy varies from $69 \%$ to $98 \%$ depending on the experience of examiner [1]. Also, intrasubstance tears cannot be evaluated in arthroscopy. MRI provides excellent soft tissue contrast and is capable of evaluating the soft tissue and bony structures in multiple imaging planes which provide significant advantages over other imaging techniques. MRI has also been demonstrated as a cost effective technique by reducing unnecessary surgical and arthroscopic interventions. Improved diagnostic accuracy has been clearly demonstrated and MRI is shown to result in changes in patient management in $41 \%$ of patients [2]. In this article, we were going to discuss about MRI findings of 120 patients who were diagnosed to have acute internal derangement of knee.

\section{Method}

This is a prospective study of hundred and twenty patients who underwent MRI for internal derangement of knee. The study period was January 2013 to January 2015. All the patients gave history of knee joint pain following trauma and clinically suspected to have meniscal and ligament tears. Patients were evaluated using Philips intera R $111.5 \mathrm{~T}$ with pulsar gradient system using a sensor extremity coil. The patient population consisted of 111 males and 9 females. Ethics committee clearance was obtained and all the patients were clearly explained in their own language and informed consent was also obtained. Patients were in the age group of 20 to 50 years. The inclusion criteria were history of twisting injuries with or without contact mechanism with knee joint pain. The exclusion criteria were Degenerative arthritis, Infections, Neoplasm and any previous surgery to the knee.

The knee joint was examined in sagittal, coronal and axial planes with T1, T2 and PD sequences. Imaging was done using an FOV of 160, $512 \times 512$ matrix and $3 \mathrm{~mm}$ slice thickness. An axial acquisition through the patella femoral joint was used as the initial localizer for subsequent coronal and sagittal plane images. The sagittal plane is primarily used to evaluate the cruciate ligaments, menisci and synovial anatomy as the coronal plane optimally evaluates the collateral ligament and body of menisci. Patient is placed in supine position with the knee externally rotated $15^{\circ}-20^{\circ}$ and flexed $5^{\circ}-10^{\circ}$. This position increases and facilitates the evaluation of $\mathrm{ACL}$ and patella femoral compartment.

\section{Result}

Majority of the patients who were diagnosed as internal derangement of knee were having anterior cruciate ligament tear and medial meniscal tear when evaluated using Mri as evident from Table 1. Lateral meniscal injury were not so common than the medial meniscal tear. We found 15 cases of ACL injuries to coexist with medial meniscus injuries $(27 \%)$. Other associated injuries were seen in medial collateral ligament $(22 \%)$ and lateral meniscus (16\%). There was a increased frequency of occurrence of MCL injury in patients with coexisting ACL injuries (Table 2).

\section{Discussion}

The major advantage of MRI is its non-invasive and non-ionizing nature. Although arthroscopy is considered the gold standard, improved diagnostic accuracy with MRI has been clearly demonstrated [3]. The study done by Ruwe et al. demonstrated that MRI reduced unnecessary arthroscopic interventions in 53 of 103 patients [4]. Another study by Vincent et al. reported that MRI reduces the need for arthroscopy in 42 patients. Majority of the patients in our study were male 111 patients and their common cause of injury was contact sports followed by road traffic accidents. Patients in the age group 20 - 30 years accounted for maximum number of cases (Table 1).

Singh et al. showed MRI to be superior to arthroscopy for diagnosing meniscal and cruciate ligament tears. The internal structure of menisci which could not be demonstrated by arthroscopy can be visualized by MRI. Also MRI can visualize peripheral meniscal tears and inferior surface tears which is the Achilles heel of arthroscopy. Another conclusion drawn from the study was the superior sensitivity of MRI in the detection of multiple meniscal tears [5]. The study by Reicher et al. reported better patient compliance and preference for MRI over arthroscopy [6]. The major pitfalls in MR imaging of knee are due to anatomic variants and artefacts, especially partial volume effects. The study by Rubin et al. reported a fall in diagnostic specificity for ligamental tears with multiple ligament injuries [7]. 
Table 1. Distribution of patients by age.

\begin{tabular}{ccc}
\hline Age & Number & Percentage \% \\
\hline $20-30$ & 66 & $55 \%$ \\
$31-40$ & 39 & $33 \%$ \\
$41-50$ & 15 & $12 \%$ \\
\hline Table 2. MRI findings. & Number & \\
\hline Cases & 54 & Percentage $\%$ \\
\hline ACL tears & 15 & $45 \%$ \\
PCL tears & 39 & $12.5 \%$ \\
Medial meniscus tear & 27 & $32 \%$ \\
Lateral meniscus tear & 6 & $22 \%$ \\
Discoid meniscus & 27 & $5 \%$ \\
Medial collateral sprain/tear & 12 & $22.5 \%$ \\
Lateral collateral sprain/tear & & $10 \%$ \\
\hline
\end{tabular}

Magnetic resonance (MR) imaging is sensitive and specific in the diagnosis of internal derangements of the knee [8] [9]. In our study of Internal Derangement of Knee, we studied 120 patients of which 48 were left knee joint and 72 were right knee joint. Forty five percentages of our cases showed ACL tears (Table 2 and Figure 1). This was the most common ligament injury in our study. This was comparable to the study by Shetty et al. who reported $37 \%$ of knee injuries to the ACL [10]. Hyper intensity of the ligament was the most common sign (55\%). $25 \%$ showed discontinuity and $16 \%$ of ligaments was not visualized which corresponded with Gentali et al. study [11]. This was also comparable to study by Shetty et al. who reported hyper intensity as the most common sign observed followed by discontinuity and lastly non visualization.

We had thirteen percentages of cases having posterior cruciate ligament tear which was more than that which was reported by Shetty and colleagues, who reported $4.5 \%$ PCL tears. Berquist reported between $2 \%-23 \%$ of tears to be PCL tears [3]. Berquist found the primary findings in PCL tears to be similar to the ACL tears except that the cause of ligament tear is not as useful. Hyper intensity was seen in 3 cases and discontinuity of ligaments in 2 cases.

The main findings in collateral ligament injuries were adjacent fascial oedema and increased signal intensity. Schweitzer et al. reported a higher frequency of MCL injury than ACL injury [12] which we could not corroborate in our study. This could be because certain sports activities (skiing) have a very high incidence of MCL injuries which are not a part of our sports culture. The incidence of medial meniscus tear is more common than lateral meniscus tears (Figure 2) which correlated with the study by Berg et al. [13]. Grade 3 lesions were the most common (45\%) subset of meniscal tears. We found 6 cases $(5 \%)$ of discoid meniscus in the lateral meniscus. This corresponds to the report by Berquist et al. who found discoid meniscus to be more common in the lateral meniscus compared to medial meniscus. Our findings also correspond to the report by Shetty et al. who found $5 \%$ incidence for discoid meniscus.

Bone contusions (Figure 3) showed a high association with ACL tears $(50 \%)$, and PCL tears $(60 \%)$. This corresponds to the study by Remer et al. who found upto $68 \%$ bone injury associated with ACL injury [14]. Mc Cauley et al. also reported a high incidence of bone bruise with ACL tears [15]. We found 15 cases of ACL injuries to coexist with medial meniscus injuries (Table 3 and Figure 4). Other associated injuries were seen in medial collateral ligament (22\%) and lateral meniscus (16\%). This corresponds to the report by Remer et al. who observed $68 \%$ of meniscal tears to be associated with ACL injuries. He also reported an increased frequency of occurrence of MCL injury in patients with coexisting ACL injuries (18\%). 


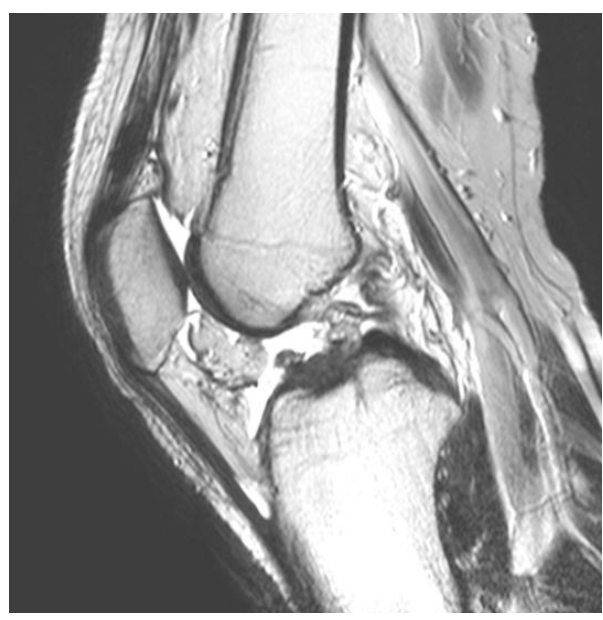

Figure 1. T1 weighted image shows joint effusion and nonvisualisation of ACL.

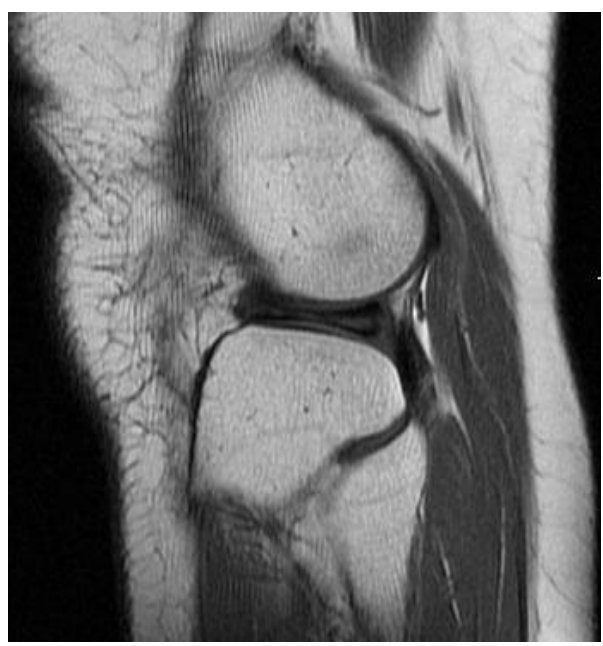

Figure 2. Stir sequence shows associated bone bruise of tibia.

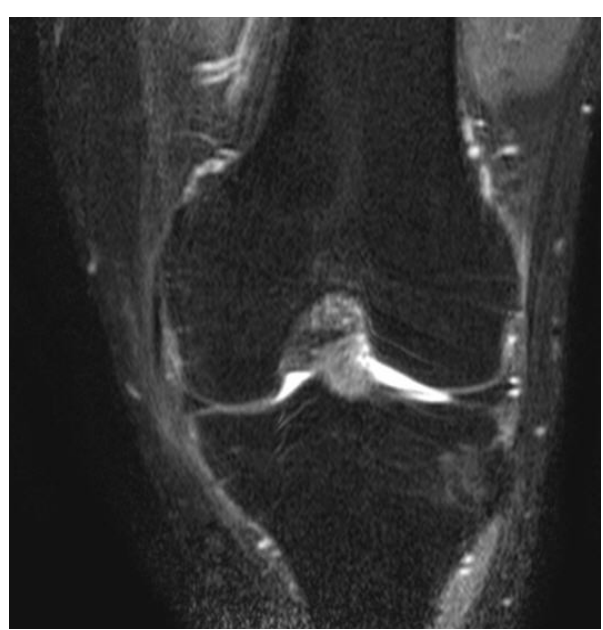

Figure 3. Grade 2 linear signal intensity in posterior horn of lateral meniscus. 


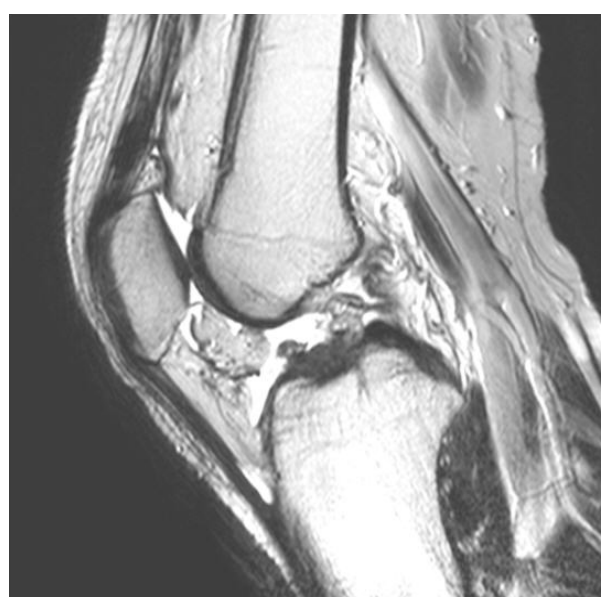

Figure 4. T2 weighted sequences showing discontinuity and hyperintensity of ACL with grade 3 meniscal tear of the posterior horn of medial meniscus.

Table 3. Multiple Ligament injuries.

\begin{tabular}{rcccccc}
\hline & ACL & PCL & MM & LM & MCL & LCL \\
\hline ACL & -- & 9 & 15 & 9 & 18 & 3 \\
PCL & 9 & -- & 9 & -- & 9 & -- \\
MM & 15 & 9 & -- & 3 & 12 & 3 \\
LM & 9 & -- & 3 & -- & 3 & 6 \\
MCL & 18 & 9 & 12 & 3 & 3 & - \\
LCL & 3 & -- & 3 & 6 & 3 \\
\hline
\end{tabular}

Note: ACL—Anterior cruciate ligament; PCL—Posterior cruciate ligament; MM—Medial meniscus; LM-Lateral meniscus; MCL—Medial collateral ligament; LCL_- Lateral collateral ligament.

\section{Conclusion}

Patients in the age group 20 - 30 years accounted for a maximum number of cases. Commonest lesion detected in our study was ACL tear followed by meniscal tear and medial collateral ligament injury. We found increased incidence of medial meniscal and collateral ligament tears associated with ACL tears. Majority of posterior cruciate ligament tears were associated with anterior cruciate, medial collateral and medial meniscus injuries. The most common sign of cruciate ligament injury was hyper intensity in the ligament. Grade 3 was the most common grade of meniscal tear. Bone bruise and anterior tibial subluxation showed a higher frequency with ACL injury. MRI is unique in its ability to evaluate the internal structure as well as the surface of the meniscus. Many anatomical variants can mimic a tear on MRI. MRI is an excellent non invasive modality for imaging the knee.

\section{References}

[1] Bredella, M.A., Tirman, P.F.J. and Peterfy, C.G. (1999) Accuracy of T2 Weighted Fast Spin Echo MR Imaging with fat Saturation in Detecting Cartilage Defects in the Knee: Comparison with Arthroscopy in 130 Patients. American Journal of Roentgenology, 172, 1073-1080. http://dx.doi.org/10.2214/ajr.172.4.10587150

[2] Maurer, E.J., Kaplan, P.A. and Dussault, R.G. (1997) Acutely Injured Knee: Effect of MR Imaging on Diagnostic and Therapeutic Decision. Radiology, 204, 799-805. http://dx.doi.org/10.1148/radiology.204.3.9280263

[3] Berquist, T.H. (2006) MRI of Musculoskeletal System. 5th Edition, Lippincott Williams and Wilkins, Philadelphia, 303-423.

[4] Ruwe, P.A., Wright, J. and Randall, R.L. (1992) Can MR Imaging Effectively Replace Diagnostic Arthroscopy? Radi- 
ology, 183, 335-339. http://dx.doi.org/10.1148/radiology.183.2.1561332

[5] Singh, J.P., Garg, L., Shrimali, R., Setia, V. and Gupta, V. (2004) MR Imaging of the Knee with Arthroscopic Correlations in Twisting Injuries. Indian Journal of Radiology Imaging, 14, 33-40.

[6] Reicher, M.A., Bassett, L.W. and Gold, R.H. (1985) High-Resolution Magnetic Resonance of the Knee Joint: Pathological Correlation. AJR, 145, 903-909. http://dx.doi.org/10.2214/ajr.145.5.903

[7] Rubin, D.A., Kettering, J.M., Towers, J.D. and Britton, C.A. (1998) MR Imaging of Knees Having Isolated and Combined Ligament Injuries. American Journal of Roentgenology, 190, 1207-1213. http://dx.doi.org/10.2214/ajr.170.5.9574586

[8] Fritz, R.C. (2003) MR Imaging of Meniscal and Cruciate Ligament Injuries. Magnetic Resonance Imaging Clinics of North America, 11, 283-293. http://dx.doi.org/10.1016/S1064-9689(03)00028-X

[9] Jung, J.Y., Yoon, Y.C. and Kwon, J.W. (2009) Diagnosis of Internal Derangement of the Knee at 3.0-T MR Imaging: 3D Isotropic Intermediate-weighted versus 2D Sequences. Radiology, 253, 781-787.

[10] Shetty, D.S., Lakshar, V.N. and Krishna, G.K. (2002) Magnetic Resonance Imaging in Pathological Conditions of Knee. Indian Journal of Radiology and Imaging, 12, 375-381.

[11] Gentili, A., Seeger, L.L., Yao, L. and Do, H.M. (1994) Anterior Crutiate Ligament Tear: Indirect Signs at MR Imaging. Radiology, 193, 835-840. http://dx.doi.org/10.1148/radiology.193.3.7972834

[12] Schweitzer, M.E., Tran, D., Deely, D.M. and Hume, E.L. (1995) Medial Collateral Ligament Injuries: Evaluation of Multiple Signs, Prevalence and Location of Associated Bone Bruises, and Assessment with MR Imaging. Radiology, 194, 825-829. http://dx.doi.org/10.1148/radiology.194.3.7862987

[13] Berg, B.C.V., Malghem, J., Poilvache, P., Maldague, B. and Lecouvet, F.E. (2005) Meniscal Tears with Fragments Displaced in Notch and Recesses of Knee: MR Imaging with Arthroscopic Comparison. Radiology, 234, 842-850. http://dx.doi.org/10.1148/radiol.2343031601

[14] Remer, E.M., Fitzgerald, S.W., Friedman, H., Rogers, L.F., Hendricks, R.W. and Schafer, M.F. (1992) Anterior Cruciate Ligament Injury: MR Imaging Diagnosis and Patterns of Injury. Radiographics, 12, 901-915. http://dx.doi.org/10.1148/radiographics.12.5.1529133

[15] McCauley, T.R., Moses, M., Kier, R., Lynch, J.K., Barton, J.W. and Jokl, P. (1994) MR Diagnosis of Tears of Anterior Cruciate Ligament of the Knee: Importance of Ancillary Finding. American Journal of Roentgenology, 162, 115-119. http://dx.doi.org/10.2214/ajr.162.1.8273648 